\title{
Thromboprophylaxis and maternal-fetal outcomes of women with serum markers for hereditary thrombophilia and previous obstetric complications
}

Cristiane Munaretto Ferreira 1

Ernesto Antonio Figueiró-Filho 2

Vanessa Marcon de Oliveira 3

Érica Freire de Vasconcelos Pereira 4

${ }_{1}$ Programa de Pós-Graduação em Saúde e Desenvolvimento da Região Centro-Oeste. Faculdade de Medicina. Universidade Federal de Mato Grosso do Sul - UFMS. Código postal 549. Cidade Universitária. Vila Ipiranga. Campo Grande, MS, Brasil. CEP: 79070-900. E-mail: cristianemunaretto@gmail.com

2 Departamento de Gynecologya e Obstetricia. Faculdade de Medicina. Universidade Federal de Mato Grosso do Sul - UFMS. Campo Grande, MS, Brasil.

3,4 Faculdade de Farmácia. Universidade Federal de Mato Grosso do Sul - UFMS. Campo Grande, Brasil.

\begin{abstract}
Objectives: to evaluate the maternal-fetal outcomes of thromboprophylaxis with enoxaparin based on scoring system in women with serum markers for hereditary thrombophilia and previous obstetric complications.

Methods: a retrospective study was undertaken based on data collected from clinical records. We included 54 pregnant women with serum markers for hereditary thrombophilia undergoing therapeutic intervention with enoxaparin in the period from November 2009 to December 2013. The initial dose of low molecular weight heparin was guided by a scoring system. The maternal-fetal outcomes of previous pregnancies and, subsequently, the treatment were compared using the chi-square $\left(\chi^{2}\right)$ test with the Yates correction and Fisher's Exact Test; $p<0.05$ was considered significant.

Results: we observed significant reduction in fetal/perinatal deaths $(p<0.05)$ and spontaneous abortions $(p<0.001)$ after intervention. The live births at full-term delivery $(p<0.001)$ and live births at preterm delivery $(p<0.05)$ increased significantly after intervention.

Conclusions: the therapeutic intervention with enoxaparin based on scoring system during pregnancy seems to improve the fetal prognosis.

Key words Thrombophilia, Pregnancy outcome, Heparin low molecular weight, Enoxaparin
\end{abstract}




\section{Introduction}

Hereditary thrombophilia is associated with approximately $50 \%$ of thromboembolic events and is listed as a risk factor for the occurrence of pregnancy complications, significantly increasing the maternal and fetal risks, including early and late pregnancy loss, placental abruption, growth restriction fetal and preeclampsia.1,2

Although there is consistent evidence that therapy with low molecular weight heparin (LMWH) reduces the occurrence of adverse obstetric outcomes in women with acquired thrombophilia, ${ }^{3}$ the benefits of this therapy in women with hereditary thrombophilia are less conclusive. ${ }^{4}$ The results regarding the efficacy of LMWH for preventing obstetric complications in this group of patients are conflicting in consequence of the range of different methodologies applied. 5 Most of these variations in methods are due to different inclusion criteria and definitions of poor pregnancy outcomes. 6 The routine use of anticoagulation in the setting of inherited thrombophilia is considered experimental. 7 The necessity of placebo controlled trials should be advocated, however, logistic and ethical difficulties limit such approach. 8

Due to dilemmas in the literature about how to act and possibly intervene in situations involving pregnant and postpartum women with inherited thrombophilia, the present study was performed to evaluate the maternal-fetal outcomes following intervention with enoxaparin based on a non-validated scoring system 8 in women with serum markers of hereditary thrombophilia and obstetric complications within whose previous pregnancies.

\section{Methods}

A retrospective study with data collected from the clinical records of women undergoing the Local Assistance Protocol for thrombophilia in pregnancy from the Clinic of Gynecology and Obstetrics ${ }^{9}$ of Faculty of Medicine of Federal University of Mato Grosso do Sul (FAMED/UFMS) Brazil, was carried out from November 2009 to December 2013.

This study included pregnant women whose pregnancies were complicated by at least one of the following options: recurrent miscarriages (two or more previous losses up to the $20^{\text {th }}$ week of pregnancy), fetal/perinatal death, and/or preeclampsia; all had at least one serum marker for hereditary thrombophilia in the current pregnancy; classified by the Scoring System proposed by Sarig et al., 8 prior to therapeutic intervention; and started used enoxa- parin previously to the $20^{\text {th }}$ week of pregnancy and correctly followed the local clinical protocol for thrombophilia. Fetal/perinatal death was considered as intrauterine death between the $20^{\text {th }}$ week of pregnancy and perinatal death within the first 7 days of life. Preeclampsia was defined as a blood pressure of $140 / 90 \mathrm{mmHg}$ or higher after 20 weeks of pregnancy, which was accompanied by proteinuria of $0.3 \mathrm{~g}$ or more in a 24 -hour urine collection. The scoring system proposed by Sarig et al., 8 is composed of four major categories: obstetrical history, previous thromboembolic events, family history and type of thrombophilia. Based on the score obtained by the sum of the categories, it is possible to highlight pregnant women's risk level low (score 5), intermediate (score 6-10), high (score 11-14) and extremely high (score 15) - and get the corresponding dose of LMWH for thromboprophylaxis of patients, ranging from 20 to $80 \mathrm{mg} /$ day. 8

The exclusion criteria were: previous presence of anticardiolipin antibodies (IgG and $\operatorname{IgM}$ ), lupus anticoagulant or anti- $\beta 2$ glycoprotein I, previous systemic lupus erythematosus, previous diagnosis of gestational diabetes mellitus, a prior episode of arterial or venous thrombosis and/or those patients that have followed a specific protocol for thrombophilia in previous pregnancies.

The presence of markers for hereditary thrombophilia was identified by levels of proteins $\mathrm{C}$ and $\mathrm{S}$, antithrombin, homocysteine and screening of the Q506 mutation of factor V (factor V Leiden). The laboratory investigation was performed at the first prenatal consultation of the current pregnancy prior to $20^{\text {th }}$ week of gestation.

The plasma level of protein $\mathrm{C}$ was performed by the chromogenic method 10,11 and values lower than $65 \%$ of activity were considered as deficiency of protein $\mathrm{C}$. The plasma determination of protein $\mathrm{S}$ was conducted using the chromogenic method, considering disability values under $30 \%$ of activity. 10,11 For measuring antithrombin, the method chosen was the cromogenic, 10,11 which was considered deficiency when values were lower than $60 \%$ of activity.

The values of plasma homocysteine were determined using a chemiluminescence - competitive immunoassay, and results higher than $12 \mu \mathrm{mol} / 1$ were considered hyperhomocysteinemia. ${ }^{12}$ The search for the factor $\mathrm{V}$ Leiden mutation was carried out using the polymerase chain reaction (PCR) according to the read restriction enzyme MNLI, considering the presence of a homozygous positive or heterozygous mutation. 10,11

According to the Local Assistance Protocol for 
thrombophilia in pregnancy, ${ }^{9}$ all pregnant women with serum markers of hereditary thrombophilia received enoxaparin from antepartum to the sixth week postpartum. The medication was discontinued at the beginning of labor when spontaneous or 24 hours prior to termination of pregnancy when planned. Pregnant women with hyperhomocysteinemia were additionally supplemented with folic acid (15 mg/day) and vitamins B6 and B12 at doses of 10 and $0.1 \mathrm{mg} /$ day, respectively. 13

The initial dose of LMWH was guided by a Scoring System proposed by Sarig et al., 8 and defined according to clinical judgment. When the scoring system indicated initial therapeutic doses, between 60 and $80 \mathrm{mg} / \mathrm{day}$, we started with doses of 40 or $60 \mathrm{mg}$ /day LMWH (clinical judgment) and as needed to adjust the actual risk. In cases of dose changing, we considered the highest dosage during pregnancy.

The information in this study was obtained from reviewing the medical records of pregnant women who met the inclusion criteria. The maternal-fetal variables studied were the following: number of miscarriages, number of fetal/perinatal deaths, number of live births with preterm delivery (birth at fewer than 37 completed weeks of pregnancy), number of live births with full-term delivery $(\geq 37$ completed weeks of pregnancy) and the occurrence of preeclampsia. The control variables were: maternal age, gestational age, previous pregnancies and ethnicity. Statistical analysis was based on the counting of the absolute number of pregnancies and comparing the maternal-fetal outcomes before and after treatment with enoxaparin.

The software package SPSS version 10 (SPSS, Chicago, IL, USA) was used to perform the statistical analysis. Chi-square test with the Yates correction was used to evaluate the association between the maternal-fetal variables. Fisher exact test was applied when appropriate. Data are presented as the relative risk (RR) with a confidence interval of $95 \%$ (CI95\%). $P$-values $<0.05$ were considered statistically significant.

This study was approved by the Research Ethics Committee, Federal University of Mato Grosso do Sul, under protocol number 1586 as of October 29, 2009, Certificate of Presentation for Ethical Consideration number: 15330913.6.0000.0021.

\section{Results}

A total of 54 patients met the inclusion criteria and had their records analyzed. We evaluated 182 pregnancies, of which 128 were previous from diagnosis and therapeutic intervention for thrombophilia and 54 were undergoing follow-up with the Local Assistance Protocol for thrombophilia in pregnancy. ${ }^{9}$ The majority of pregnant women in the study were Caucasian (57.4\%), with a mean age of $30.74 \pm 6.09(18-46)$ years in the current pregnancy, and had an average of $2.37 \pm 1.29$ (1 - 6) pregnancies prior to therapeutic intervention.

Table 1 shows the maternal-fetal outcomes of pregnancies before and after the intervention with LMWH. The frequency of live births increased from $32.0 \%(41 / 128)$ to $96.3 \%(52 / 54)(p<0.001)$ after therapeutic intervention with enoxaparin. In these women, the number of fetal/perinatal deaths and miscarriages decreased significantly. Additionally, there were significantly more live births with fullterm delivery than pregnancies without LMWH treatment. However, the occurrence of preeclampsia between pregnancies with and without treatment with LMWH did not reach statistical significance.

Based upon the scoring system proposed by Sarig et al., $824.1 \%$ (13/54) of the pregnancies were at low risk, $51.8 \%(28 / 54)$ intermediate risk, $20.4 \%$ $(11 / 54)$ high risk and $3.7 \%(2 / 54)$ extremely high risk.

Of fifty-four pregnancies treated, 74.1\% (40/54) used only LMWH, and $25.9 \%$ (14/54) used LMWH combined with folic acid and B vitamins. The dose of $20 \mathrm{mg}$ /day of LMWH was used in $25.9 \%(14 / 54)$ of pregnancies, $40 \mathrm{mg} /$ day in $76.2 \%(34 / 54)$ and 60 $\mathrm{mg} /$ day in $1.9 \%(1 / 54)$ of cases.

\section{Discussion}

The management of thrombophilia during pregnancy and postpartum frequently involves anticoagulant therapy. The potential beneficial effect of LMWH in preventing obstetric complications in women with hereditary thrombophilia has been partially extrapolated from the documented efficacy in cases of antiphospholipid antibody syndrome.4,5 However, the results on the role of LMWH during pregnancy have substantial heterogeneity. ${ }^{14}$

Although the mechanisms that explain the beneficial effects of LMWH are not fully understood, it is suggested that beyond its anticoagulation action, heparin can inhibit activation of the complement system, decrease vascular resistance and modulate trophoblastic invasion by stimulating specific activity of proteases, such as matrix metalloproteinases, or reducing the epithelial cell adhesion complex, such as protein E-cadherin. Abnormal expression of E-cadherin protein has been related to the history of fetal loss and recurrent 


\begin{tabular}{|c|c|c|c|c|c|c|}
\hline & \multicolumn{4}{|c|}{ Therapeutic intervention for thrombophilia } & \multirow[t]{3}{*}{$p$} & \multirow[t]{3}{*}{ RR (Cl95\%) } \\
\hline & \multicolumn{2}{|c|}{ Before $(N=128)$} & \multicolumn{2}{|c|}{ After $(\mathrm{N}=54)$} & & \\
\hline & $\mathrm{n}$ & $\%$ & $\mathrm{n}$ & $\%$ & & \\
\hline \multicolumn{7}{|l|}{ Miscarriage } \\
\hline Present & 63 & 49.2 & 0 & - & $<0.001 * *$ & _- \\
\hline Absent & 65 & 50.8 & 54 & - & & \\
\hline \multicolumn{7}{|l|}{ Fetal/perinatal death } \\
\hline Present & 24 & 18.7 & 2 & 3.7 & $<0.05 * *$ & $0.20(0.05-0.81)$ \\
\hline Absent & 104 & 81.3 & 52 & 96.3 & & \\
\hline \multicolumn{7}{|l|}{ Preeclampsia } \\
\hline Present & 18 & 14.1 & 7 & 13.0 & ns & $0.92(0.41-2.07)$ \\
\hline Absent & 110 & 85.9 & 47 & 87.0 & & \\
\hline Live births & & & & & $<0.001 *$ & \\
\hline Present & 41 & 32.0 & 52 & 96.3 & & $7.70(4.38-13.5)$ \\
\hline Absent & 87 & 68.0 & 2 & 0.7 & & \\
\hline Full-term delivery ( $G A \geq 37$ weeks) & & & & & $<0.001 *$ & \\
\hline Present & 29 & 22.6 & 39 & 72.2 & & $2.80(1.99-3.92)$ \\
\hline Absent & 99 & 77.4 & 15 & 27.8 & & \\
\hline Preterm birth (GA < 37weeks) & & & & & $<0.001 *$ & \\
\hline Present & 12 & 9.4 & 13 & 24.1 & & $2.57(1.25-5.26)$ \\
\hline Absent & 116 & 90.6 & 41 & 75.9 & & \\
\hline
\end{tabular}

LMWH =low molecular weight heparin; * $\chi^{2}$ with Yates correction; ** F is her exact test. GA = gestational age; R R = relative ris $\mathrm{k} ; \mathrm{Cl}=$ confidence interval; $\mathrm{ns}=$ not significant.

miscarriages. 15,16

The probability of fetal loss decreases significantly with the use of LMWH.17 This premise was reinforced by present study because the use of enoxaparin during pregnancy has prevented the occurrence of spontaneous abortions and significantly reduced the number of fetal/perinatal deaths.

The reports of successful pregnancy with the use of anticoagulant therapy in women with hereditary thrombophilia and fetal loss, previous history of preeclampsia and other complications range from 60.0 to $93.0 \% .2,18$ These results are comparable to those described in our study, wherein $96.3 \%$ of the cases had live births after intervention with enoxaparin. Term births reached $72.2 \%$ of the treated pregnancies, indicating that thromboprophylaxis with enoxaparin sodium increased the number of live births at term 2.8-fold $(\mathrm{RR}=2.80 ; \mathrm{CI} 95 \%=1.99$ 3.92).

Despite treatment, $24.1 \%$ of live births were preterm deliveries. It is likely that individual patient factors, such as maternal age, risk behaviors during pregnancy, the type of complications in previous pregnancies, and the presence of infection or hypertensive comorbidities that led to therapeutic interruption of pregnancy, influenced the frequency of this outcome.18,19 The percentage of preterm cases in our study infants was similarly verified by Elmahashi et al. 20 According to these authors, labor occurred spontaneously in most cases; however, in some cases, there was premature induction due to the presence of preeclampsia and intrauterine growth restriction. 20

Preeclampsia is a major cause of maternal fetal morbidity and mortality. In women with a previous episode, the risk of recurrence in subsequent pregnancies is important, ranging from $25.0 \%$ to $65.0 \% .{ }^{21}$ In agreement with the literature, our study observed the recurrence of severe preeclampsia in $27.8 \%(5 / 18)$ of the patients. Unfortunately, it was not possible to confirm the effects of LMWH on the risk of preeclampsia. Although the effect of LMWH in reducing the risk of preeclampsia is expected due to the reduction in the formation of thrombosis and anti-apoptotic effect on trophoblasts, it is important to note that the risk of preeclampsia is mainly influenced by other characteristics from the patients, 
such as hypertension, diabetes mellitus, underlying renal disease, high body mass index, age and previous episodes of preeclampsia. ${ }^{22,23}$

There are still no definitive conclusions about the optimal diagnostic and treatment approaching for hereditary thrombophilia in pregnancy. 5,24 Some guidelines do not support screening for serum markers of hereditary thrombophilia unless they are present prior to a personal or family history of thromboembolism. 5,24 However, many obstetrical services consider clinically useful for investigating the presence of hereditary thrombophilia in women with a history obstetric complications. ${ }^{25,26}$ In this study, most of the women had at least one fetal loss over 20 weeks of pregnancy or at least two consecutive miscarriages. In this context, tracking markers for hereditary thrombophilia was considered beneficial. The individualized approach for cases, based on the type of thrombophilic defect, family history and presence of additional risk factors, is essential for clinical decisions about whether or not to initiate anticoagulant therapy. ${ }^{8}$ Additionally, it is important to consider investigating thrombophilia in order to define more precisely the impact this condition has on adverse obstetric outcomes, as well as determine the best evidence-based intervention.

Finally, the limitations of this study include the small sample size due to the low prevalence of some thrombophilic defects and the inability to measure the presence of a mutation in the methylentetrahydrofolate reductase gene and prothrombin due to the high cost of this test for the

\section{References}

1. De Stefano V, Martinelli I, Rossi E, Battagliole T, Za T, Mannuccio MP, Leone G. The risk of recurrent venous thromboembolism in pregnancy and puerperium without antithrombotic prophylaxis. Br J Haematol. 2006; 135: 38691.

2. Kosar A, Kasapoglu B, Kalyoncu S, Turan H, Balcik OS, Gümüs EI. Treatment of adverse perinatal outcome in inherited thrombophilias: a clinical study. Blood Coagul Fibrinolysis. 2011; 22: 14-8.

3. Hoppe B, Burmester GR, Dorner T. Heparin or aspirin or both in the treatment of recurrent abortions in women with antiphospholipid antibody (syndrome). Curr Opin Rheumatol. 2011; 23: 299-304.

4. Rambaldi MP, Mecacci F, Guaschino S, Paidas MJ. Inherited and Acquired Thrombophilias. Reprod Sci. 2014; 21: 167-82.

5. Bates SM, Greer IA, Middeldorp S, Veenstra DL, Prabulo AM, Vandvik PO. Venous thromboembolism, thrombophilia, antithrombotic therapy, and pregnancy:
Brazilian Health System. Regarding the study's retrospective nature, although recognized as a limitation, pregnant women with adverse obstetric histories are unwilling to be randomly distributed in studies with pharmacological intervention or comparison groups. ${ }^{17}$ Therefore, the inclusion of results from several pregnancies for the same woman in the analysis offers advantages to counter selection bias and allows for ideal matching of maternal genetic factors, age and obstetric history. 27 Nevertheless, the present study contributes to the small number of actual studies reported in the literature that evaluate treatment with LMWH in pregnant women with thrombophilia and adverse obstetric outcomes, even though it might be a low grade level of evidence, due to the study design. There are randomized clinical trials questioning the ability of LMWH to reduce adverse pregnancy outcomes. However, the relatively low number of women with thrombophilia compromises beneficial results in this group of patients.6,28,29

In conclusion, women with serum markers for hereditary thrombophilia and previous obstetric complications may benefit from treatment with enoxaparin in terms of increasing the number of live births. Despite the Scoring System proposed by Sarig et al.,8 has not been validated, it was particularly useful for guiding the initial dose of enoxaparin administered during pregnancy. In the patients studied herein, however, the prescribed dose was determined by clinical judgment.
Antithrombotic Therapy and Prevention of Thrombosis, 9th ed: American College of Chest Physicians Evidence-Based Clinical Practice Guidelines. Chest. 2012; 141 (Suppl. 2): e691S-736S.

6. Mutlu I, Mutlu MF, Biri A, Erdem M, Erdem A. Effects of anticoagulant therapy on pregnancy outcomes in patients with thrombophili a and previous poor obstetric history. Blood Coagul Fibrinolysis. 2015: 26 (3): 267-73.

7. Rodger MA, Carrier M, Le Gal G, Martinelli I, Perna A, Rey E, de Vries JI, Gris JC. Meta-analysis of low-molecular-weight heparin to prevent recurrent placenta-mediated pregnancy complications. Blood. 2014; 123 (6): 822-8.

8. Sarig G, Vidergor G, Brenner B. Assessment and management of high-risk pregnancies in women with thrombophilia. Blood Rev. 2009; 23: 143-7.

9. Figueiro-Filho EA, Oliveira VM, Breda I, Coelho LR, Ferreira CM. [Usefulness of a scoring system on perinatal outcomes in pregnant women with thrombophilia in the effectiveness of an enoxaparin-based intervention]. Rev Bras Ginecol Obstet. 2012; 34 (10): 459-65. 
10. Walker ID, Greaves M, Preston FE. Haemostasis and thrombosis task force, British Committee for standards in haematology. Investigation and management of heritable thrombophilia. Br J Haemato. 2001; 114 (3): 512-28.

11. Jackson BR, Holmes K, Phansalkar A, Rodger GM. Testing for hereditary thrombophilia: a retrospective analysis of testing referred to a national laboratory. BMC Clin Pathol 2008; 8: 3 .

12. Stauffenberg MT, Lange RA, Hillis D, Cigarroa J, Hsu RM, Devaraj S, Jialal I. Hyperhomocysteinemia measured by immunoassay: a valid measure of coronary artery atherosclerosis. Arch Pathol Lab Med. 2008; 128 (11): 1263-6.

13. Brazão ML, Silva AS, Gaspar J, Barros C, Pereira H, Arauújo JN. [Thrombophilia and recurrent miscarriages]. Medicina Interna. 2010; 17 (4): 191-9.

14. D'Ippolito S, Ortiz AS, Veglia M, Tersigni C, Di Simone N. Low molecular weight heparin in obstetric care: a review of the literature. Reprod Sci. 2011; 18 (7): 602-13.

15. Erden O, Imir A, Guvenal T, Muslehiddinoglu A, Arici S, Cetin M, Cetin A. Investigation of the effects of heparin and low molecular weight heparin on E-cadherin and laminin expression in rat pregnancy by immunohistochemistry. Hum Reprod. 2006; 21 (11): 3014-8.

16. Mulla MJ, Myrtolli K, Brosens JJ, Chamley LW, KwakKin JY, Paidas MJ, Abrahams VM. Antiphospholipid antibodies limit trophoblast migration by reducing IL-6 production and STAT3 activity. Am J Reprod Immunol. 2010; 63 (5): 339-48.

17. Kovac M, Mikovic Z, Mitic G, Djordjevic V, Mandic V, Rakicevic L, Radojkovic D. Does Anticoagulant Therapy Improve Pregnancy Outcome Equally, Regardless of Specific Thrombophilia Type? Clin Appl Thromb Hemost. 2013; 20 (2) :184-9.

18. Bittar RE, Fonseca EB, Zugaib M. [Prediction and prevention of pretem delivery]. Femina. 2010; 38 (1): 13-22.

19. Araujo BF, Tanaka AC. [Risk factors associated with very low birth weight in a low-income population]. Cad Saúde Pública. 2007; 23 (12): 2869-77.
20. Elmahashi MO, Elbareg AM, Essadi FM, Ashur BM, Adam I. Low dose aspirin and low-molecular-weight heparin in the treatment of pregnant Libyan women with recurrent miscarriage. BMC Res Notes. 2014; 7:23. Avaliable: http://www.biomedcentral.com/content/pdf/1756-0500-723.pdf via the INTERNET. Accessed June 11, 2014.

21. Cudihy D, Lee RV. The pathophysiology of pre-eclampsia: current clinical concepts. J Obstet Gynaecol. 2009; 29 (7): 576-82.

22. Duckitt K, Harrington D. Risk factors for pre-eclampsia at antenatal booking: systematic review of controlled studies. BMJ. 2005; 330 (7491): 565. Avaliable: http://www.bmj.com/content/bmj/330/7491/565.full.pdf via the INTERNET. Accessed June 11, 2014

23. Pabinger I. Thrombophilia and its impact on pregnancy. Thromb Res. 2009; 123 (Suppl. 3): S16-S21.

24. Riva E. [Hereditary thrombophilia: association with venous thromboembolic disease and obstetric complications]. Arch Med Int. 2011; 33 (supl. 2): S3-S5.

25. Hoffmann E, Hedlund E, Perin T, Lyndrup J. Is thrombophilia a risk factor for placenta-mediated pregnancy complications? Arch Gynecol Obstet. 2012; 286 (3): 585-9.

26. Davenport WB, Kutteh WH. Inherited thrombophilias and adverse pregnancy outcomes: a review of screening patterns and recommendations. Obstet Gynecol Clin North Am. 2014; 41 (1): 133-44

27. Leduc L, Dubois E, Takser L, Rey E, David M. Dalteparin and low-dose aspirin in the prevention of adverse obstetric outcomes in women with inherited thrombophilia. J Obstet Gynaecol Can. 2007; 29 (10): 787-93.

28. Clark P, Walker ID, Langhorne P, Crichton L, Thomson Greaves M, Whyte S, Greer IA. SPIN (Scottish Pregnancy Intervention) study: a multicenter, randomized controlled trial of low-molecular-weight heparin and low-dose aspirin in women with recurrent miscarriage. Blood. 2010; 115 (21): 4162-7

29. Visser J, Ulander VM, Helmerhorst FM, Lampinen K, Morin Papunen L, Bloemenkamp KW, Kaaja RJ. Thromboprophylaxis for recurrent miscarriage in women with or without thrombophilia. Habenox: a randomised multicentre trial. Thromb Haemost. 2011; 105 (2): 295-301.

Received on March 16, 2017

Final version presented on September 12, 2017

Approved on October 3, 2017 